\title{
Osteogenesis Imperfecta: An Uncommon Association with Non-Communicating Hydrocephalus
}

\author{
Chatterjee $\mathrm{K}^{1}$, Dolui $\mathbf{S}^{2}$, Das $\mathbf{S}^{3}$, Paul $\mathrm{A}^{4}$ \\ ${ }^{1}$ Dr. Kaushani Chatterjee, MBBS, MD Resident, \\ Calcutta Medical College, ${ }^{2} \mathrm{Dr}$. Sanat Dolui, MBBS, MD, \\ Assistant Professor, Calcutta National Medical College \\ and Hospital, ${ }^{3} \mathrm{Dr}$. Suman Das, MBBS, MD Resident, \\ B C Roy Children Hospital, ${ }^{4}$ Dr. Ayan Paul MBBS, \\ MD Resident. Calcutta National Medical College and \\ Hospital, Kolkata.
}

\section{Introduction}

$\mathrm{O}$ steogenesis imperfecta (OI) or Brittle bone disease, the most common genetic cause of osteoporosis, is caused by structural or quantitative defect in type 1 collagen. Ol has a triad of fragile bone, blue sclera and early deafness ${ }^{1}$. Hydrocephalus may rarely be associated, more so with the perinatally lethal type 2 OI. Here we present a case of type $3 \mathrm{OI}$ with noncommunicating hydrocephalus and patent foramen ovale.

\section{The Case}

An eight day old male baby, born of $1^{\text {st }}$ degree consanguineous marriage, was admitted in our hospital with inability to move both the lower limbs and excessive cry on handling. The mother also complained of poor suck and yellowish discolouration of skin and sclera along with swelling of both lower limbs. The baby was delivered vaginally at full term by breech extraction. There is no history of similar disease in any of the family members \& no history of any foetal/sibling loss.

On examination, the baby weighed $2.25 \mathrm{~kg}$ and had swelling of both the thighs that were extremely tender. There was inward curvature of both legs below the knees. General survey revealed icterus with fullness of anterior fontanelle and sutural diastasis, with a head

\begin{abstract}
Osteogenesis imperfecta (OI) is a generalised disorder of connective tissue presenting with fragile bones, blue sclera and early deafness. Hydrocephalus is usually associated with type $2 \mathrm{OI}$. Here we present a rare case of noncommunicating hydrocephalus and patent foramen ovale in type 3 OI.
\end{abstract}

circumference of $37 \mathrm{~cm}$. Eye examination showed blue discolouration of the sclera.

Investigations revealed : Hb- $13.6 \mathrm{~g} / \mathrm{dl}, \mathrm{TC}-17,500 /$ cmm (N-72,L-28), ESR-12mm/hr, TSB-12.2mg/dl, serum calcium, phosphate $\&$ alkaline phosphatase were all within normal limits ruling out rickets, renal tubular acidosis \& hypoparathyroidism. CSF showed 5 cells, all lymphocytes with $159 \mathrm{mg} / \mathrm{dl}$ protein \& normal sugar \& chloride. $X$ ray showed $B / L$ undisplaced fracture femur with bending of tibia \& fibula. CXR showed long and narrow thorax but the baby did not have any respiratory problems. Blood culture showed no growth, USG brain revealed hydrocephalus with enlargement of lateral and third ventricles and CT scan showed noncommunicating hydrocephalus with periventricular ooze and effaced basal cisterns. Echocardiography showed patent foramen ovale and BAER testing showed B/L moderate hearing loss.

The baby was initially managed with IV antibiotics due to suspected sepsis that was later omitted after the negative reports on culture \& CSF analysis. He was given oral calcium \& vit. D supplementation and the femur fractures were managed by the orthopaedic surgeon. Currently he is on regular follow up for any increase in head circumference that might need neurosurgical intervention in the future. Hydrocephalus. J Nepal Paediatr Soc 2013;33(1):63-65. 


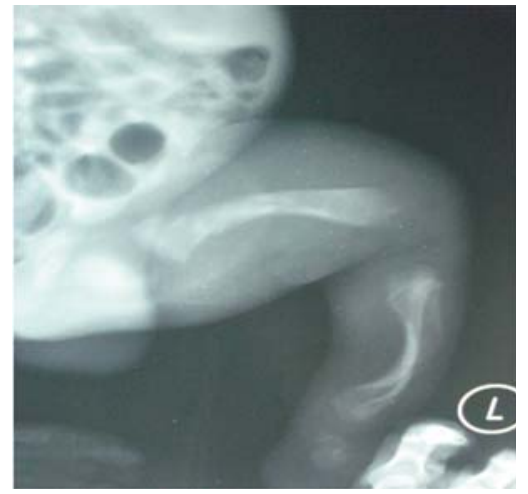

Fig 1: Undisplaced fracture femur with bending of tibia and fibula.

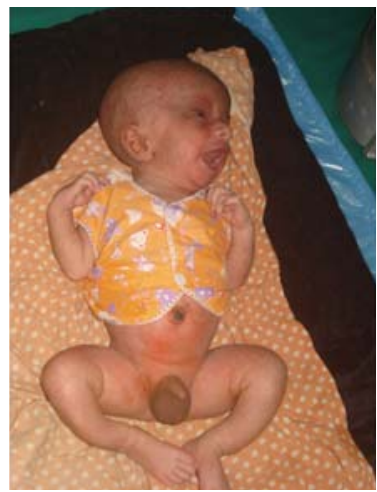

Fig 2: The baby with swelling of bilateral thighs and bending of tibia and fibula of both legs.

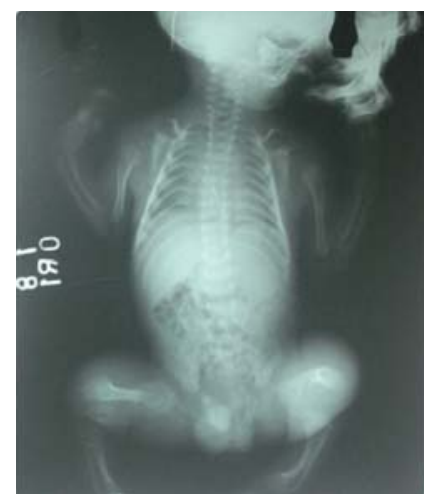

Fig 3: Babygram showing long and narrow thorax.

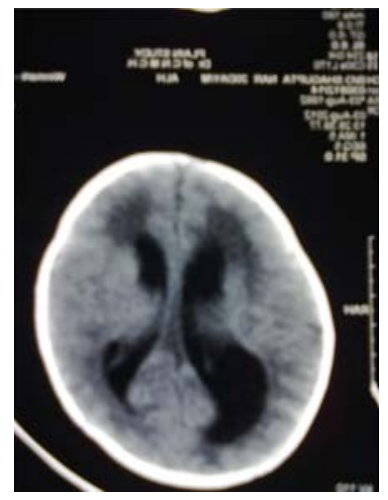

Fig 4: CT scan brain shows non communicating hydrocephalus with periventricular ooze.

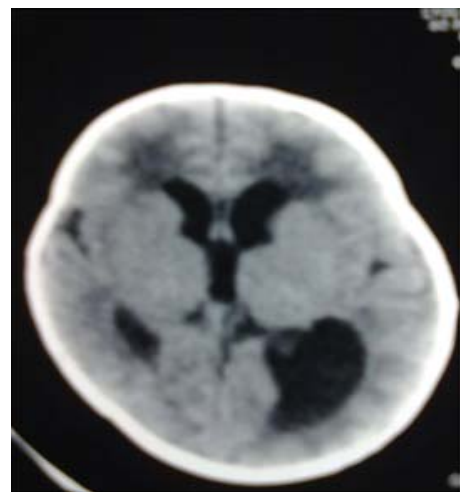

Fig 5: CT scan brain shows non communicating hydrocephalus with periventricular ooze.

\section{Discussion}

Ol is autosomal dominant disorder with a frequency of 1 in 20,000 cases being detectable in infancy. The collagen structural mutations cause OI bone to be globally abnormal. The bone matrix contains abnormal type I collagen fibrils and relatively increased levels of type III and V collagen. In addition, several noncollagenous proteins are also reduced ${ }^{1}$.

The Sillence Classification divides OI into 4 types based on clinical and radiological criteria, additional types have been proposed based on histologic distinctions. Ol was once divided into 'congenita' and 'tarda' depending whether the forms were detectable at birth or in later childhood ${ }^{1}$.

Types of $\mathrm{Ol}$ presenting with fractures at birth encompasses type 2 (perinatal lethal), type 3 (progressive deforming) and type 4 (moderately severe). Type 2 OI have multiple fractures at birth with a crumpled appearance with striking micromelia and multiple rib fractures with beading. Type $3 \mathrm{OI}$ apart from having in-utero fractures, have relative macrocephaly, triangular facies and disorganised bone matrix giving a popcorn appearance at metaphyses, along with scoliosis and vertebral compression. Type 4 Ol may present with in-utero fractures along with bowing of lower leg bones. Like type $1 \mathrm{OI}$, individuals of type 4 Ol are divided into types $A$ and $B$ based on presence/ absence of dentinogenesis imperfecta ${ }^{2}$.

The recognised neurological complications of OI include craniovertebral junction abnormalities like basilar invagination, syringohydromyelia, hydrocephalus and brainstem compression ${ }^{3}$. Hydrocephalus, usually of the communicating variety, is found in type $2 \mathrm{OI}$. Our case shows the association of noncommunicating hydrocephalus in type $3 \mathrm{OI}$ - the proposed mechanism being in-utero vascular compromise due to associated craniovertebral junction anomalies ${ }^{4}$. This child also presented with patent foramen ovale which is also a rare association. 
Scleral hue in Scillence classification ranges from blue in type 1 to white in type 4; the bluish tinge may result from decreased sclera thickness ${ }^{5}$. Other causes of blue sclera includes Marshall-Smith syndrome, Roberts-SC Phocomelia, Russell-Silver syndrome, also occasionally in Aarskog syndrome, Ehler-Danlos and Marfan syndromes, hypophosphatasia, incontinentia pigmenti and others ${ }^{6}$.

The differential diagnosis of severe OI includes thanatotropic dysplasia, achondrogenesis type 1, campomelic dysplasia; type 3 Ol needs to be differentiated from infantile hypophosphatasia. Hypophosphatasia results in low serum alkaline phosphatase (ALP) level and increased serum inorganic phosphate while in this case the serum ALP level is normal as seen in OI.

In autosomal dominant OI, a severe presentation is likely to be the result of a spontaneous mutation that occurred at or around conception, the affected individual is likely to be the first affected person in the family.

Treatment with bisphosphonates is effective in improving mobility and decreasing symptoms in many patients. Intravenous pamidronate or oral alendronate improve quality of life and inhibit bone resorption thus increasing bone mineralisation ${ }^{7,8}$.

\section{Conclusion}

Communicating hydrocephalus is commonly associated with type 2 OI. Bluish colouration of the sclera can occur with normal scleral thickness due to tissues with different proteoglycan compositions and hence hydration that reflects wavelengths of colour. This case highlights the importance of follow up of patients with $\mathrm{OI}$ with annual MRI and proper physical rehabilitation and early ambulation.

\section{References}

1. Marini JC. Osteogenesis Imperfecta; In : Kliegman RM, Jenson HB, Behrman R, Stanton BS edited : Nelson Textbook of Pediatrics, Vol 2,18 ${ }^{\text {th }}$ ed; Philadelphia; Saunders, Elsevier; 2007;pg-28872890.

2. Levin LS, Salinas CF, Jorgenson RJ. 1978 Classification of osteogenesis imperfecta by dental characteristics. Lancet 1978;1:332-333.

3. Ramirez N, Vilella FE, Colon M, Flynn JM. Osteogenesis imperfecta and hyperplasic callus formation in a family: a report of three cases and review of literature. J Pediatr Ortho 2003;12:88-96.

4. Paterson CR, McAllion S, Miller R. Osteogenesis imperfecta with dominant inheritance and normal sclerae. J Bone Joint Surg Br 1983;65:35-9.

5. Sillence D, Butler B, Latham M, Barlow K 1993 Natural history of blue sclerae in osteogenesis imperfecta. Am J Med Genet 45:183-186.

6. Jones $\mathrm{KL}$, Osteogenesis Imperfecta syndrome types 1 and 2, In: Jones KL edited: Smith's Recognizable Patterns of Human Malformation; Philadelphia, Saunders, Elsevier, 2009, 6 $6^{\text {th }}$ ed, pg562-563.

7. Astrom E, Soderhall S. Beneficial effect of longterm intravenous biphosphonate in Osteogenesis imperfecta. Arch Dis Child 2002;86:356-64.

8. Plotkin $\mathrm{H}$, Rauch $\mathrm{F}$. Pamidronate treatment in severe osteogenesis imperfecta in children under 3 years age. J Clin Endocrinol Metab 2000;11:85-90. 